PROCEEDINGS OF THE

AMERICAN MATHEMATICAL SOCIETY

Volume 126, Number 3, March 1998, Pages 637-644

S $0002-9939(98) 04427-X$

\title{
RATIONAL CURVES ON K3 SURFACES IN $\mathbb{P}^{1} \times \mathbb{P}^{1} \times \mathbb{P}^{1}$
}

\author{
ARTHUR BARAGAR
}

(Communicated by Ron Donagi)

\begin{abstract}
We discuss Manin and Batyrev's notion of the arithmetic stratification of a variety, and, for an irreducible surface $V$ embedded in $\mathbb{P}^{m}$, compare it with the spectrum of degrees of rational curves on $V$. We study this spectrum for the class of K3 surfaces generated by smooth $(2,2,2)$ forms in $\mathbb{P}^{1} \times \mathbb{P}^{1} \times \mathbb{P}^{1}$.
\end{abstract}

\section{INTRODUCTION}

In this paper, we study the spectrum of degrees of rational curves on varieties in the class of $\mathrm{K} 3$ surfaces generated by smooth $(2,2,2)$ forms in $\mathbb{P}^{1} \times \mathbb{P}^{1} \times \mathbb{P}^{1}$. We show in Theorem 2.1 that generic and non-generic varieties of this type are distinguished by this spectrum. On a generic variety of this type, there is an action of a group of automorphisms $\mathcal{A} \cong \mathbb{Z} / 2 * \mathbb{Z} / 2 * \mathbb{Z} / 2$ generated by $\sigma_{1}, \sigma_{2}$ and $\sigma_{3}$. We define a vector height $\mathbf{h}$ on rational curves in $V$ with the property $\operatorname{deg} C=(1,1,1) \cdot \mathbf{h}(C)$, and show in Theorem 3.1 that there exist linear maps $T_{i}$ such that $\mathbf{h}\left(\sigma_{i} C\right)=T_{i} \mathbf{h}(C)$. Thus, if we can find a rational curve $C$ in $V$, and can measure $\mathbf{h}(C)$, then this can be used to find a large subset of the spectrum of degrees of rational curves of $V$. In particular, if $C$ is a rational fiber with respect to a projection onto one of the components of $\mathbb{P}^{1} \times \mathbb{P}^{1} \times \mathbb{P}^{1}$, then the degree of $C$ is either 2 or 4 , and in Theorem 4.1 we show that the number of rational curves on $V$ in the $\mathcal{A}$-orbit of $C$ with degree less than $T$ is $(2 / \pi \sqrt{3} \operatorname{deg} C) T+O(\sqrt{T} \log T)$.

\section{The ARITHMETIC STRATIFICATION OF A VARIETY}

We begin with a discussion of Batyrev and Manin's arithmetic stratification of a variety $[\mathrm{B}-\mathrm{M}]$.

Let $V=V(K)$ be a variety, defined over a number field $K$, and, for simplicity, embedded in a projective space $\mathbb{P}^{m}$. Let $H$ be the usual universal exponential height on $\mathbb{P}^{m}$. Let $U$ be a subset of $V$, and define

$$
N_{U}(T)=\#\{P \in U: H(P)<T\} .
$$

Associated to $U$, we can define a zeta function

$$
Z_{U}(s)=\sum_{P \in U}(H(P))^{-s} .
$$

Received by the editors May 9, 1996 .

1991 Mathematics Subject Classification. Primary 14J28, 14J50, 14 G05.

(c)1998 American Mathematical Society 
There exists an $\alpha_{U} \in \mathbb{R}$ such that $Z_{U}(s)$ converges for all $s$ with $\operatorname{Re}(s)>\alpha_{U}$ and diverges if $\operatorname{Re}(s)<\alpha_{U}$. We call $\alpha_{U}$ the exponent of $N_{U}(T)$, since it is related to $N_{U}(T)$ by the well known result (for divergent $N_{U}(T)$ )

$$
\limsup _{T \rightarrow \infty} \frac{\log N_{U}(T)}{\log T}=\alpha_{U}
$$

Note that $\alpha_{U}$ depends on the number field $K$. For example, if $V=K$, then $\alpha_{V}=2[K: \mathbb{Q}]$. Let us normalize this exponent by setting $\beta_{U}=\alpha_{U} /[K: \mathbb{Q}]$. This is the exponent of $N_{U}(T)$ with respect to the non-universal height $\hat{H}=H^{[K: \mathbb{Q}]}$. It may still be the case that $\beta_{U}$ depends on $K$ (for example, let $V$ be an elliptic curve with rank 0 over $\mathbb{Q}$ and positive rank over $K$ ), but the hope is that $\beta_{U}$ is constant for all number fields over some sufficiently large $K$.

We define the arithmetic stratification $S_{V}$ of $V$ to be:

$$
\begin{aligned}
S_{V} & =\left\{\beta_{U}: U \text { is a non-empty Zariski open subset of } V\right\} \\
& \subset \mathbb{R}^{+} \cup\{0,-\infty\} .
\end{aligned}
$$

Let

$$
\hat{\beta}_{V}=\inf S_{V}
$$

Then for any $\epsilon>0$, there exists a Zariski closed subset $W(\epsilon)$ of $V$ so that

$$
N_{V}(T)=N_{W(\epsilon)}(T)+a(T) T^{\hat{\beta}_{V}},
$$

where

$$
a(T)=\left\{\begin{array}{c}
O\left(T^{\epsilon}\right), \\
\Omega\left(T^{-\epsilon}\right) .
\end{array}\right.
$$

The constants implied by the big $O$ and $\Omega$ depend on $\epsilon$. If $S_{V} \neq\left\{\hat{\beta}_{V}\right\}$, then the first term dominates the asymptotic behaviour of $N_{V}(T)$ for $\epsilon$ sufficiently small. Since $W(\epsilon)$ is a proper subvariety of $V$, we often dismiss this term as a characterization of $W(\epsilon)$ and not $V$. The arithmetic stratification of $V$ is a crude measure of the possibilities for $W(\epsilon)$, but is more accurately a reflection of the characteristics of $V$.

We can completely characterize $S_{V}$ for curves. We note that $V$ is a finite union of irreducible curves, so let us first assume $V$ is irreducible. If $V$ has genus $g \geq 2$, then it has a finite number of rational points, so $S_{V}=\{-\infty\}$. If $V$ has genus $g=1$, then $S_{V}=\{0\}$ if the rank of $V$ is positive, and $S_{V}=\{-\infty\}$ otherwise. If $V$ is an irreducible rational curve embedded in $\mathbb{P}^{m}$, then there exist coprime polynomials $X_{i}(t) \in K[t]$ so that the map

$$
t \mapsto\left(X_{0}(t), \ldots, X_{m}(t)\right)
$$

is a map of $K$ onto all but finitely many points in $V$. The degree of $V$ is the maximum of the degrees of the polynomials $X_{i}(t)$, and $S_{V}=\left\{\beta_{V}\right\}=\{2 / \operatorname{deg} V\}$. Returning now to $V$ an arbitrary curve, we see that $S_{V}$ is always finite.

If the dimension of $V$ is at least 2, then it may be possible that $S_{V}$ is infinite. In the following sections we exhibit such an example, provided Manin and Batyrev's conjecture [B-M, Conjecture A] is true (that is, provided $\hat{\beta}_{V} \leq 0$ ).

Suppose $\beta_{U}$ is greater than $\beta_{U^{\prime}}$ and both are in $S_{V}$. Then there exists a Zariski closed subset $W$ in $V$ (equal to the closure of $U \cup U^{\prime} \backslash U \cap U^{\prime}$ ) such that $\beta_{W}=\beta_{U}$. Thus, we can instead consider the spectrum

$$
S_{V}^{*}=\left\{\beta_{W}: W \text { is a Zariski closed proper subset of } V\right\} .
$$


Note that either $\beta_{V} \notin S_{V}^{*}$, in which case $S_{V}=\left\{\beta_{V}\right\}$; or $\beta_{V} \in S_{V}^{*}$ and $S_{V} \subset S_{V}^{*}$. In the latter case, we also have either

$$
S_{V}=\left\{\beta \in S_{V}^{*}: \beta>\hat{\beta}_{V}\right\}
$$

or

$$
S_{V}=\left\{\beta \in S_{V}^{*}: \beta \geq \hat{\beta}_{V}\right\} .
$$

Note also that since $W$ is a finite union of proper irreducible subvarieties of $V$, there exists an irreducible subvariety $W^{\prime}$ in $W$ so that $\beta_{W^{\prime}}=\beta_{W}$. Thus we may characterize $S_{V}^{*}$ as

$$
S_{V}^{*}=\left\{\beta_{W}: W \text { is a proper irreducible subvariety of } V\right\} .
$$

Let us now suppose $V$ is an irreducible variety of dimension two. If $W$ is a non-empty proper irreducible subvariety of $V$, then $W$ is a curve or point, and as we saw earlier, if $\beta_{W}>0$, then $W$ is a rational curve. Thus, for an irreducible surface $V$,

$$
S_{V}^{*}=\{2 / \operatorname{deg} W: W \text { is an irreducible rational curve in } V\} \cup S_{0},
$$

where $S_{0}$ is either $\{0,-\infty\}$ or $\{-\infty\}$. In particular,

$$
S_{V} \subset\{2 / n: n \in \mathbb{Z}\} \cup\{0,-\infty\} .
$$

Thus, if $V$ is a surface and $\hat{\beta}_{V}>0$, then $S_{V}$ is finite. This gives us some information about three dimensional hypersurfaces: If $V$ is a variety of dimension three, then $S_{V}$ has at most one cluster point and that point is its infimum $\hat{\beta}_{V}$.

In this vein, Batyrev and Manin wonder whether $S_{V}$ is finite for every Fano variety [B-M, Question 1.10]. More generally, one might wonder whether it is ever possible for a cluster point of $S_{V}$ to have a value other than zero.

For a variety $V$ with trivial canonical divisor, Batyrev and Manin conjecture [B-M, Conjecture A]

$$
\hat{\beta}_{V}=\inf S_{V} \leq 0 .
$$

In the following, we study a class of surfaces with trivial canonical divisor.

\section{A FAMily of K3 SURfaces}

Let $V$ be a smooth variety generated by a $(2,2,2)$ form in $\mathbb{P}^{1} \times \mathbb{P}^{1} \times \mathbb{P}^{1}$. Then $V$ is the zero locus of a non-singular polynomial over $K$ in three projective variables which is quadratic in each variable. That is to say, if we write $(X, Y, Z) \in \mathbb{P}^{1} \times$ $\mathbb{P}^{1} \times \mathbb{P}^{1}$, where $X=\left(X_{0}: X_{1}\right)$, etc., then $V$ is the zero locus of a non-singular polynomial $F$ of the form

$$
F(X, Y, Z)=\sum_{i_{k}+j_{k}=2} a_{i_{1} j_{1} i_{2} j_{2} i_{3} j_{3}} X_{0}^{i_{1}} X_{1}^{j_{1}} Y_{0}^{i_{2}} Y_{1}^{j_{2}} Z_{0}^{i_{3}} Z_{1}^{j_{3}}
$$

with coefficients in $K$. This variety is a K3 surface, and hence its canonical divisor is trivial.

We can write

$$
F(X, Y, Z)=X_{0}^{2} F_{0}(Y, Z)+X_{0} X_{1} F_{1}(Y, Z)+X_{1}^{2} F_{2}(Y, Z) .
$$


The equations $F_{i}=0$ describe three curves in $\mathbb{P}^{1} \times \mathbb{P}^{1}$, which generically do not intersect (over $\overline{\mathbb{Q}}$.) If there is no common point of intersection, then for all $P=$ $\left(P_{1}, P_{2}, P_{3}\right) \in V$, the equation

$$
F\left(X, P_{2}, P_{3}\right)=0
$$

is quadratic in $X$, and since $P_{1} \in \mathbb{P}^{1}(K)$ is a solution, the other root $P_{1}^{\prime}$ is in $\mathbb{P}^{1}(K)$. Thus, we can define a rational automorphism of $V$,

$$
\sigma_{1}:\left(P_{1}, P_{2}, P_{3}\right) \mapsto\left(P_{1}^{\prime}, P_{2}, P_{3}\right) .
$$

In a similar fashion, there exist three curves associated to each of the $Y$ and $Z$ components. We call $V$ generic within this family of K3 surfaces if none of these three sets of three curves have a common point of intersection over $\overline{\mathbb{Q}}$.

Since we have defined the notion of degree for rational curves in terms of an embedding into a projective space $\mathbb{P}^{m}$, let us embed $\mathbb{P}^{1} \times \mathbb{P}^{1} \times \mathbb{P}^{1}$ into $\mathbb{P}^{7}$ via the Segre embedding:

$$
\phi:(X, Y, Z) \mapsto \quad \begin{aligned}
\left(X_{0} Y_{0} Z_{0}:\right. & X_{0} Y_{0} Z_{1}: X_{0} Y_{1} Z_{0}: X_{1} Y_{0} Z_{0} \\
& \left.: X_{0} Y_{1} Z_{1}: X_{1} Y_{0} Z_{1}: X_{1} Y_{1} Z_{0}: X_{1} Y_{1} Z_{1}\right) .
\end{aligned}
$$

We define the degree of $C$ to be $\operatorname{deg}(\phi(C))$.

Now suppose $C$ is an irreducible rational curve in $V$. Then there exist polynomials $X_{i}(t), Y_{i}(t), Z_{i}(t) \in K[t]$ so that $X_{0}(t)$ and $X_{1}(t)$ are coprime, etc., and the map

$$
\mathbf{X}(t)=\left(\left(X_{0}(t): X_{1}(t)\right),\left(Y_{0}(t): Y_{1}(t)\right),\left(Z_{0}(t): Z_{1}(t)\right)\right)
$$

is a map from $K$ onto all but finitely many points of $C$. Define the height

$$
h_{X}(C)=\max \left\{\operatorname{deg} X_{0}(t), \operatorname{deg} X_{1}(t)\right\}
$$

and define $h_{Y}$ and $h_{Z}$ similarly. Note that the height $h_{X}(C)$ is well defined, even though the polynomials $X_{0}(t)$ and $X_{1}(t)$ are not necessarily uniquely defined. Now define the vector height

$$
\mathbf{h}(C)=\left(h_{X}(C), h_{Y}(C), h_{Z}(C)\right) .
$$

Then

$$
\operatorname{deg}(C)=h_{X}(C)+h_{Y}(C)+h_{Z}(C)=(1,1,1) \cdot \mathbf{h}(C) .
$$

Theorem 2.1. Let $V$ be in the family of $K 3$ surfaces generated by smooth $(2,2,2)$ forms in $\mathbb{P}^{1} \times \mathbb{P}^{1} \times \mathbb{P}^{1}$. For such a $V$, the following are equivalent:

1) $V$ is generic within this family of $K 3$ surfaces.

2) $2 \notin S_{V}^{*}$ for any number field $L$ over $K$.

3) $\phi(V)$ contains no lines over $\overline{\mathbb{Q}}$.

Proof. Suppose $V$ is not generic within this family of $\mathrm{K} 3$ surfaces. Then there exists a common point of intersection in $\overline{\mathbb{Q}}$ for one of the three sets of curves described above. For ease, let us assume that point is $\left(P_{2}, P_{3}\right) \in \mathbb{P}^{1} \times \mathbb{P}^{1}$ over a number field $L$ and that $F_{i}\left(P_{2}, P_{3}\right)=0$ for $i=1,2$ and 3 . Then $F\left(X, P_{2}, P_{3}\right)=0$ for all $X \in \mathbb{P}^{1}$, which describes a curve $C$ in $V$ with $\mathbf{h}(C)=(1,0,0)$. Thus, $2 \in S_{V}^{*}$, and since $\operatorname{deg} C=1, \phi(C)$ is a line in $\phi(V)$.

Note that there exists a rational curve $C$ over $L$ with $\beta_{C}=2$ if and only if $\phi(C)$ is a line. Suppose such a $C$ exists. Then $\operatorname{deg} C=1$, so $\mathbf{h}(C)=(1,0,0),(0,1,0)$ or 
$(0,0,1)$. Let us assume without loss of generality that $\mathbf{h}(C)=(1,0,0)$. Then there exists a map

$$
t \mapsto(X(t), Y, Z)
$$

from $L$ onto almost all of $C$ with $Y$ and $Z$ constant. This is possible only if $F_{i}(Y, Z)=0$ for all $i$. That is, such a $C$ exists only if $V$ is not generic.

\section{Generic SURfaCES In THIS FAMILY}

If $V$ is generic within this family, then associated to each of the components $X, Y$ and $Z$, there is a rational automorphism $\sigma_{1}, \sigma_{2}$ and $\sigma_{3}$. The group $\mathcal{A}$ of automorphisms generated by $\sigma_{1}, \sigma_{2}$ and $\sigma_{3}$ is isomorphic to $\mathbb{Z} / 2 * \mathbb{Z} / 2 * \mathbb{Z} / 2$ [W2] (see also $[\mathrm{B}]$.)

Theorem 3.1. Suppose $C$ is an irreducible rational curve on $V$. Then $\sigma_{i} C$ is an irreducible rational curve on $V$ and

$$
\mathbf{h}\left(\sigma_{i} C\right)=T_{i} \mathbf{h}(C),
$$

where

$$
T_{1}=\left[\begin{array}{ccc}
-1 & 2 & 2 \\
0 & 1 & 0 \\
0 & 0 & 1
\end{array}\right], \quad T_{2}=\left[\begin{array}{ccc}
1 & 0 & 0 \\
2 & -1 & 2 \\
0 & 0 & 1
\end{array}\right], \quad T_{3}=\left[\begin{array}{ccc}
1 & 0 & 0 \\
0 & 1 & 0 \\
2 & 2 & -1
\end{array}\right] .
$$

Proof. We show this for $\sigma_{1}$. Note that $\sigma_{1}$ fixes $Y$ and $Z$, so $h_{Y}\left(\sigma_{1} C\right)=h_{Y}(C)$ and $h_{Z}\left(\sigma_{1} C\right)=h_{Z}(C)$. Let

$$
\sigma_{1}(X(t), Y(t), Z(t))=\left(X^{\prime}(t), Y(t), Z(t)\right) .
$$

Then, by considering Eq. (1) affinely, we have for $F_{0} X_{1} X_{1}^{\prime} \neq 0$

$$
\frac{X_{0}^{\prime}(t) X_{0}(t)}{X_{1}^{\prime}(t) X_{1}(t)}=\frac{F_{2}(Y(t), Z(t))}{F_{0}(Y(t), Z(t))}
$$

Recall that $X_{0}$ and $X_{1}$ are coprime, so $X_{0}$ divides $F_{2}$ and $X_{1}$ divides $F_{0}$ (see (1)). Thus, there exists a polynomial $m(t) \in K[t]$ so that

$$
m X_{0}^{\prime}=\frac{F_{2}}{X_{0}} \quad \text { and } \quad m X_{1}^{\prime}=\frac{F_{0}}{X_{1}} .
$$

If $m$ is not a constant, then there exists a $t_{0} \in \overline{\mathbb{Q}}$ so that $m\left(t_{0}\right)=0$. Then $F_{0}\left(Y\left(t_{0}\right), Z\left(t_{0}\right)\right)=F_{0}\left(t_{0}\right)=0$ and $F_{2}\left(t_{0}\right)=0$. If $X_{0}\left(t_{0}\right) \neq 0$ and $X_{1}\left(t_{0}\right) \neq 0$, then we must have $F_{1}\left(t_{0}\right)=0$, which means $V$ is not generic. Thus, we may assume $X_{0}\left(t_{0}\right)=0$ and $X_{1}\left(t_{0}\right) \neq 0$. Let $r \geq 1$ be the multiplicity of the root $t_{0}$ for $X_{0}$. Then $\left(t-t_{0}\right)^{r+1}$ divides $F_{2}$, and hence it also divides $-X_{0}^{2} F_{0}-X_{1}^{2} F_{2}=X_{0} X_{1} F_{1}$. Thus, $F_{1}\left(t_{0}\right)=0$, and again we get $V$ is not generic. Thus $m(t)$ is a constant. Hence,

$$
\begin{aligned}
\operatorname{deg} X_{0}^{\prime} & =\operatorname{deg} F_{2}-\operatorname{deg} X_{0}, \\
\operatorname{deg} X_{1}^{\prime} & =\operatorname{deg} F_{0}-\operatorname{deg} X_{1} .
\end{aligned}
$$

Let us write $\mathbf{h}(C)=(n, m, l)$, and

$$
\begin{aligned}
& X_{0}(t)=a_{0} t^{n}+\text { terms of lower degree, } \\
& X_{1}(t)=a_{1} t^{n}+\ldots
\end{aligned}
$$


Since $h_{X}(C)=n$, at least one of $a_{0}$ and $a_{1}$ is non-zero. That is, $a=\left(a_{0}: a_{1}\right) \in \mathbb{P}^{1}$. Let us also write

$$
\begin{aligned}
& Y_{0}(t)=b_{0} t^{m}+\ldots \quad \text { and } \quad Y_{1}(t)=b_{1} t^{m}+\ldots, \\
& Z_{0}(t)=c_{0} t^{l}+\ldots \quad \text { and } \quad Z_{1}(t)=c_{1} t^{l}+\ldots .
\end{aligned}
$$

Since $F_{0}(Y, Z)$ is a quadratic polynomial in $Y$ and $Z$, we expect $\operatorname{deg} F_{0}(t)=2 m+2 l$. If not, then the largest powers must cancel. That is, $F_{0}(b, c)=0$.

So suppose first that $\operatorname{deg} X_{0}=\operatorname{deg} X_{1}=n$. Then $h_{X}\left(\sigma_{1} C\right) \neq 2 m+2 l-n$ only if $F_{0}(b, c)=F_{2}(b, c)=0$. But then $\operatorname{deg}\left(X_{0}^{2} F_{0}\right)<2 m+2 l+2 n$ and $\operatorname{deg}\left(X_{1}^{2} F_{2}\right)<$ $2 m+2 l+2 n$, so $\operatorname{deg}\left(X_{0} X_{1} F_{1}\right)<2 m+2 l+2 n$. That is, $\operatorname{deg} F_{1} \neq 2 m+2 l$, so $F_{1}(b, c)=0$, and $V$ is not generic.

Now suppose $n=\operatorname{deg} X_{0}>\operatorname{deg} X_{1}$. Let us write $\operatorname{deg} X_{1}=n-\epsilon$ with $\epsilon>$ 0 . If $\operatorname{deg} F_{0}=2 m+2 l$, then $\operatorname{deg}\left(X_{0}^{2} F_{0}\right)=2 m+2 l+2 n$, while $\operatorname{deg}\left(X_{0} X_{1} F_{1}\right)$ and $\operatorname{deg}\left(X_{1}^{2} F_{2}\right)$ are strictly smaller. Thus we cannot have $\operatorname{deg} F_{0}=2 m+2 l$, so $F_{0}(b, c)=0$. Let us write $\operatorname{deg} F_{0}=2 m+2 l-\delta_{1}$. Now suppose $\operatorname{deg} F_{1}=2 m+2 l$. Then

$$
\begin{aligned}
\operatorname{deg}\left(X_{0}^{2} F_{0}\right) & =2 n+2 m+2 l-\delta_{1}, \\
\operatorname{deg}\left(X_{0} X_{1} F_{1}\right) & =2 n+2 m+2 l-\epsilon, \\
\operatorname{deg}\left(X_{1}^{2} F_{2}\right) & =2 n+2 m+2 l-2 \epsilon-\delta_{2},
\end{aligned}
$$

where $\delta_{2} \geq 0$. Since these three terms add to zero, and the degree of the last is less than the degrees of the other two, those degrees must be equal. That is, $\delta_{1}=\epsilon$. Hence,

$$
\begin{aligned}
& \operatorname{deg} X_{0}^{\prime}=2 m+2 l-\delta_{2}-n \leq 2 m+2 l-n, \\
& \operatorname{deg} X_{1}^{\prime}=2 m+2 l-\epsilon-(n-\epsilon)=2 m+2 l-n,
\end{aligned}
$$

as desired.

Now suppose $F_{1}(b, c)=0$. Since we also have $F_{0}(b, c)=0$, we must have $F_{2}(b, c) \neq 0$. That is, $\operatorname{deg} F_{2}=2 m+2 l$. Thus,

$$
\begin{aligned}
\operatorname{deg} X_{0}^{\prime} & =2 m+2 l-n, \\
\operatorname{deg} X_{1}^{\prime} & =2 m+2 l-\delta_{1}-(n-\epsilon),
\end{aligned}
$$

so we must show that $\delta_{1} \geq \epsilon$. Suppose otherwise - that is, $\delta_{1}<\epsilon$. Then

$$
\begin{aligned}
\operatorname{deg}\left(X_{0}^{2} F_{0}\right) & =2 n+2 m+2 l-\delta_{1}>2 n+2 m+2 l-\epsilon, \\
\operatorname{deg}\left(X_{0} X_{1} F_{1}\right) & <2 n-\epsilon+2 m+2 l, \\
\operatorname{deg}\left(X_{1}^{2} F_{2}\right) & =2 n-2 \epsilon+2 m+2 l<2 n+2 m+2 l-\epsilon .
\end{aligned}
$$

Thus, these terms cannot cancel to give zero, so we must have $\delta_{1} \geq \epsilon$ as desired.

We leave to the reader the cases not covered by taking an affine perspective of Eq. (1).

The matrices $T_{i}$ are not surprisingly the same as were found in [B]. However, when compared to results found for rational points, the striking feature of this theorem is the absence of an error term. 


\section{ORBIts of RATIONAL FIBERS}

If we can find a rational curve $C$ in $V$, and are able to calculate $\mathbf{h}(C)$, then we can use Theorem 2.1 to find an infinite subset of $S_{V}^{*}$.

Let $C$ be a fiber on $V$ with respect to the projection onto one of the variables. We know that in $\overline{\mathbb{Q}}$, there are a finite positive number of such fibers which are singular. Such a rational fiber is the zero locus of a singular $(2,2)$ form in $\mathbb{P}^{1} \times \mathbb{P}^{1}$. One can parameterize such a curve with polynomials $Y_{i}(t)$ and $Z_{i}(t)$ of degree at most two. Thus, for any generic $V$, there exists an $L$ over $K$ so that one can find a rational curve $C$ in $V$ over $L$ with $\mathbf{h}(C)=(0, m, n)$ and $m, n \leq 2$. But since there are no curves on a generic $V$ with $\operatorname{deg} C=1$, we know $\mathbf{h}(C)=(0,1,1)$ or $(0,2,2)$ (note that if $\mathbf{h}(C)=(0,1,2)$, then $\left.\mathbf{h}\left(\sigma_{3} C\right)=(0,1,0)\right)$. Thus, over $L, S_{V}^{*}$ contains one of the two following sets:

$$
\begin{gathered}
\{1,1 / 3,1 / 7,1 / 13,1 / 19,1 / 21,1 / 31,1 / 37,1 / 39,1 / 43,1 / 49, \ldots\}, \\
\{1 / 2,1 / 6,1 / 14,1 / 26,1 / 38,1 / 42,1 / 62,1 / 74,1 / 78,1 / 86,1 / 98, \ldots\} .
\end{gathered}
$$

Counting the number of elements in the orbit of a vector under the action of the group generated by $T_{1}, T_{2}$ and $T_{3}$ is usually equivalent to a difficult lattice point problem in hyperbolic space. We are fortunate that the orbit of a vector of the form $(0, c, c)$ is an exception, and can be handled using geometric methods.

Theorem 4.1. Suppose $C$ is a rational curve on $V$ and $\mathbf{h}(C)=(0,1,1)$ or $(0,2,2)$. Then the number of rational curves on $V$ in the $\mathcal{A}$-orbit of $C$ with degree less than $T$ is

$$
\frac{2}{\pi \sqrt{3} \operatorname{deg} C} T+O(\sqrt{T} \log T)
$$

Proof. Note that

$$
\begin{aligned}
& T_{1}\left(u^{2}, v^{2},(u+v)^{2}\right)=\left((2 u+v)^{2}, v^{2},(u+v)^{2}\right), \\
& T_{2}\left(u^{2}, v^{2},(u+v)^{2}\right)=\left(u^{2},(u+2 v)^{2},(u+v)^{2}\right) .
\end{aligned}
$$

Thus, we are counting the number of coprime positive pairs $(u, v)$ which satisfy

$$
u^{2}+v^{2}+u v<\frac{T}{\operatorname{deg} C} .
$$

By Theorem 3.5 of $[\mathrm{B}]$ this is

$$
\frac{6}{\pi^{2}}|R|+O(|\partial R| \log T),
$$

where $R$ is the portion of the above ellipse in the first quadrant, and $|R|$ and $|\partial R|$ are its area and perimeter. The area of this region is

$$
\frac{T}{\sqrt{3} \operatorname{deg} C} \arctan (\sqrt{3})=\frac{\pi T}{3 \sqrt{3} \operatorname{deg} C},
$$

and the perimeter is $O(\sqrt{T})$, giving us the result.

As we noted before, if $V$ is generic in this family of K3 surfaces, then there exists a sufficiently large field $K$ so that there exists on $V(K)$ a rational curve of degree 2 or 4 . On the other hand, there is never a curve of degree 1 . Thus one might find the following quantity of interest:

$$
S_{V(\overline{\mathbb{Q}})}^{*}=\bigcup_{K} S_{V(K)}^{*}
$$


where the union is taken over all number fields $K$. It seems conceivable that there might be a sufficiently large $K$ so that $S_{V(\overline{\mathbb{Q}})}^{*}=S_{V(K)}^{*}$.

\section{ACKNOWLEDGEMENTS}

I would like to thank Steven Lu, Cam Stewart, Joe Silverman and Trevor Wooley for their encouragement and enlightenment.

\section{REFERENCES}

[B] Baragar, A., Rational points on K3 surfaces in $\mathbb{P}^{1} \times \mathbb{P}^{1} \times \mathbb{P}^{1}$, Math. Ann. 305 (1996), 541-558. MR 97g:14020

[B-M] Batyrev, V. V., Manin, Yu. I., Sur le nombre des points rationnels de hauteur borné des variétés algébriques, Math. Ann. 286 (1990), 27 - 43. MR 91g:11069

[F-M-T] Franke, J., Manin, Yu. I., Tschinkel, Yu., Rational Points of Bounded Height on Fano Varieties, Invent. Math. 95 (1989), 421 - 435; 102 (1990), 463. MR 89m:11060; MR 91i: 11068

[L] Lang, S., Number Theory III, New York: Springer Verlag 1991. MR 93a:11048

[S] Silverman, J. H., Rational Points on K3 Surfaces: A New Canonical Height, Invent. Math. 105 (1991), 347 - 373. MR 92k:14025

[T] Tschinkel, Yu., Finite Heights and Rational Points on Surfaces, Advances in Number Theory, F. Gouvea and N. Yui (eds.), Oxford University Press 1991, 319 - 329. MR 97a: 11100

[V-W] Vaughan, R. C., Wooley, T. D., On a certain nonary cubic form and related equations, Duke Math. J. 80 (1995), 669 - 735. MR 96j:11038

[W1] Wang, L., Rational Points and Canonical Heights on K3-surfaces in $\mathbb{P}^{1} \times \mathbb{P}^{1} \times \mathbb{P}^{1}$, Contemporary Math. 186 (1995), 273 - 289. MR 97a:14023

[W2] _ The Automorphism Groups of K3 Surfaces with Picard Number 3, (to appear).

Department of Mathematics, University of Waterloo, Waterloo, Ontario, Canada N2L $3 \mathrm{G} 1$

Current address: Department of Mathematical Sciences, University of Nevada, Las Vegas, Nevada 89154-4020

E-mail address: baragar@nevada.edu 